\title{
Water adsorption on vanadium oxide thin films in ambient relative humidity
}

Cite as: J. Chem. Phys. 152, 044715 (2020); https://doi.org/10.1063/1.5138959

Submitted: 16 November 2019 . Accepted: 08 January 2020 . Published Online: 31 January 2020

Dana Goodacre (D), Monika Blum, Christin Buechner (D), Harmen Hoek, Sabrina M. Gericke (D), Vedran Jovic, Joseph B. Franklin, Salinporn Kittiwatanakul (D), Tilo Söhnel (D), Hendrik Bluhm (D), and Kevin E. Smith (iD

\section{COLLECTIONS}

Paper published as part of the special topic on Oxide Chemistry and Catalysis Note: This article is part of the JCP Special Topic on Oxide Chemistry and Catalysis.

This paper was selected as Featured


\section{ARTICLES YOU MAY BE INTERESTED IN}

Understanding the effects of water on oxide catalyst surfaces

Scilight 2020, 051111 (2020); https://doi.org/10.1063/10.0000668

The catalytic nature of protein aggregation

The Journal of Chemical Physics 152, 045101 (2020); https://doi.org/10.1063/1.5133635

$\mathrm{MoO}_{3} / \mathrm{Al}_{2} \mathrm{O}_{3}$ catalysts for chemical-looping oxidative dehydrogenation of ethane

The Journal of Chemical Physics 152, 044713 (2020); https://doi.org/10.1063/1.5135920

Lock-in Amplifiers Find out more today

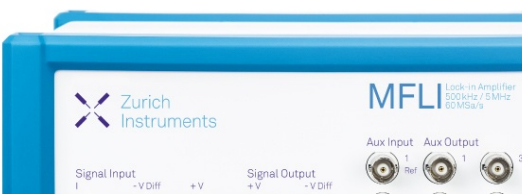

(2. (2). (1) (0). (-)
Zurich

- Instruments 


\title{
Water adsorption on vanadium oxide thin films in ambient relative humidity ${ }^{\circ}$ (ब)
}

\author{
Cite as: J. Chem. Phys. 152, 044715 (2020); doi: 10.1063/1.5138959 \\ Submitted: 16 November 2019 - Accepted: 8 January 2020 • \\ Published Online: 31 January 2020
}

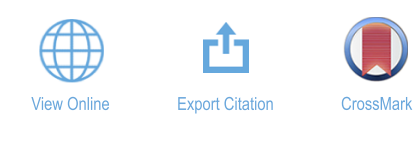

\author{
Dana Goodacre, ${ }^{1,2,3}$ (D) Monika Blum, ${ }^{2,4}$ Christin Buechner, ${ }^{4}$ (D) Harmen Hoek, ${ }^{4,5}$ Sabrina M. Gericke, \\ Vedran Jovic, ${ }^{3,7}$ Joseph B. Franklin, ${ }^{8,9}$ Salinporn Kittiwatanakul, ${ }^{10,11}$ (D) Tilo Söhnel, ${ }^{1}$ \\ Hendrik Bluhm, ${ }^{12, a)}$ (D) and Kevin E. Smith ${ }^{13, a)}$ (D)
}

\begin{abstract}
AFFILIATIONS
1' School of Chemical Sciences, University of Auckland, Auckland 1142, New Zealand

${ }^{2}$ Advanced Light Source, Lawrence Berkeley National Laboratory, Berkeley, California 94720, USA

${ }^{3}$ The MacDiarmid Institute for Advanced Materials and Nanotechnology, Wellington, New Zealand

${ }^{4}$ Chemical Sciences Division, Lawrence Berkeley National Laboratory, Berkeley, California 94720, USA

${ }^{5}$ University of Twente, Enschede, The Netherlands

${ }^{6}$ Lund University, Lund, Sweden

${ }^{7}$ National Isotope Center, GNS Science, Lower Hutt, New Zealand

${ }^{8}$ Energy Storage and Distributed Resources Division, Lawrence Berkeley National Laboratory, Berkeley, California 94720, USA

${ }^{9}$ Electrochemical Innovation Lab, Department of Chemical Engineering, University College London, London, United Kingdom

${ }^{10}$ Department of Materials Science and Engineering, University of Virginia, Charlottesville, Virginia 22904, USA

${ }^{11}$ Department of Physics, Faculty of Science, Chulalongkorn University, Bangkok, Thailand

${ }^{12}$ Fritz Haber Institute of the Max Planck Society, Department of Inorganic Chemistry, Berlin, Germany

${ }^{13}$ Boston University, Boston, Massachusetts 02215, USA
\end{abstract}

Note: This article is part of the JCP Special Topic on Oxide Chemistry and Catalysis.

a) Authors to whom correspondence should be addressed: bluhm@fhi-berlin.mpg.de and ksmith@bu.edu

\begin{abstract}
In this work, ambient pressure $\mathrm{x}$-ray photoelectron spectroscopy (APXPS) is used to study the initial stages of water adsorption on vanadium oxide surfaces. V 2p, O 1s, C 1s, and valence band XPS spectra were collected as a function of relative humidity in a series of isotherm and isobar experiments. Experiments were carried out on two $\mathrm{VO}_{2}$ thin films on $\mathrm{TiO}_{2}(100)$ substrates, prepared with different surface cleaning procedures. Hydroxyl and molecular water surface species were identified, with up to 0.5 ML hydroxide present at the minimum relative humidity, and a consistent molecular water adsorption onset occurring around $0.01 \%$ relative humidity. The work function was found to increase with increasing relative humidity, suggesting that surface water and hydroxyl species are oriented with the hydrogen atoms directed away from the surface. Changes in the valence band were also observed as a function of relative humidity. The results were similar to those observed in APXPS experiments on other transition metal oxide surfaces, suggesting that $\mathrm{H}_{2} \mathrm{O}-\mathrm{OH}$ and $\mathrm{H}_{2} \mathrm{O}-\mathrm{H}_{2} \mathrm{O}$ surface complex formation plays an important role in the oxide wetting process and water dissociation. Compared to polycrystalline vanadium metal, these vanadium oxide films generate less hydroxide and appear to be more favorable for molecular water adsorption.
\end{abstract}

Published under license by AIP Publishing. https://doi.org/10.1063/1.5138959

\section{INTRODUCTION}

Under ambient conditions, all materials on earth are exposed to water vapor, which causes water and hydroxyl layers to form on their surfaces. The role these layers play in the reactivity and electronic properties of surfaces is a subject relevant to heterogeneous catalysis, environmental science, and the operation of electronic devices. ${ }^{1-3}$ For the vast majority of materials, simple questions regarding the 
amount of adsorbed water as a function of relative humidity (RH), and the structure of the first layers in contact with the surface, remain largely unanswered.

Vanadium oxides are widely studied both for their applications in catalysis and their unique electronic properties, which make them interesting candidates for devices such as sensors and transistors. ${ }^{4,5}$ Vanadium can exist in several stable oxidation states, the most stable being the $3+, 4+$, and fully oxidized $5+$ states. The vanadium-oxygen phase diagram is rich in intermediate phases, where two oxidation states are stable in a single extended structure. Between $\mathrm{V}_{2} \mathrm{O}_{3}$ and $\mathrm{VO}_{2}$ exist the Magneli phases $\left(\mathrm{V}_{n} \mathrm{O}_{2 n-1}\right)$, which contain a mixture of vanadium $3+$ and vanadium $4+$, while between $\mathrm{VO}_{2}$ and $\mathrm{V}_{2} \mathrm{O}_{5}$ there are the Wadsley phases $\left(\mathrm{V}_{2 n} \mathrm{O}_{5 n-2}\right)$, which have a mixture of vanadium $4+$ and vanadium $5+{ }^{4,6-8}$ Each of these phases can also contain oxygen or vanadium defects, and deviate from the nominal stoichiometry. ${ }^{7,9}$ The ability of these materials to reversibly stabilize a variety of oxidation states has made them great candidates for heterogeneous catalysis. ${ }^{6} \mathrm{~V}_{2} \mathrm{O}_{5}$ is used industrially to oxidize sulfur dioxide to sulfuric acid. ${ }^{10,11}$ Oxidesupported and mixed vanadium oxides have also been applied in the reduction of nitrogen oxides in flue gas, in the selective oxidation of alkanes and alkenes, in the oxidative dehydrogenation of alkanes, and in the selective oxidation of methanol to formaldehyde. $^{10-12}$ Water is present in the atmosphere during these catalytic processes and, in many cases, is a reactant or a product. The presence of water in the atmosphere is also important to consider for electronic devices. ${ }^{3}$ It is well known that surface adsorbates and reconstruction can affect the electronic properties of vanadium oxides. $^{13-16}$

One of the most commonly used surface science techniques is $\mathrm{x}$-ray photoelectron spectroscopy (XPS). XPS allows quantitative determination of the elemental composition as well as specific chemical information about the surface constituents. Importantly, adsorbed molecular water can be distinguished from hydroxyl groups. There have been some XPS studies on the interaction of water with well-defined vanadium oxide surfaces. ${ }^{17-19}$ However, these studies have been carried out under low temperature ultrahigh vacuum conditions, which do not represent the operating environment of catalysts and electronic devices.

Ambient pressure XPS (APXPS) has made detailed surface studies in the presence of gases possible at pressures and temperatures approaching those in ambient or catalytic environments. APXPS on vanadium oxides is a subject that researchers have only just begun to explore. Water adsorption has been studied by APXPS on polycrystalline vanadium metal, ${ }^{20}$ and there have been several in situ studies on oxide-supported vanadium oxide and vanadium phosphorus oxide catalysts, ${ }^{21,22}$ but so far a more fundamental in situ study looking at water adsorption on a defined vanadium oxide surface has not been published. In this work, the adsorption of water on $\mathrm{VO}_{2}$ films is examined. In heterogeneous catalysis, vanadium oxide films or nanoparticles are frequently deposited on bulk oxide substrates such as $\mathrm{TiO}_{2}$ or $\mathrm{Al}_{2} \mathrm{O}_{3}$, which reduces cost and enhances reactivity relative to pure vanadium oxide materials. The $\mathrm{V}^{3+}, \mathrm{V}^{4+}$, and $\mathrm{V}^{5+}$ states have all been found to participate in the catalytic processes. ${ }^{23}$ It is also of interest to compare $\mathrm{VO}_{2}$ with its widely studied, structurally similar counterpart-rutile $\mathrm{TiO}_{2}$. Meanwhile, of all the vanadium oxides, $\mathrm{VO}_{2}$ has attracted the most attention for potential applications in electronic devices due to the temperature-dependent metal-insulator transition (MIT) it undergoes at near ambient temperatures. ${ }^{4,5}$ It is within this context that $\mathrm{VO}_{2}$ films on $\mathrm{TiO}_{2}$ substrates are considered a good starting point for investigation on water adsorption on vanadium oxides in general.

\section{EXPERIMENTAL}

Measurements were performed at beamline 11.0.2 of the Advanced Light Source (ALS) at Lawrence Berkeley National Laboratory using the APXPS- 1 endstation. ${ }^{24}$ These experiments were conducted on two $\sim 80 \mathrm{~nm}$ vanadium oxide films, grown on oriented rutile $\mathrm{TiO}_{2}$ (100) substrates by different methods. $\mathrm{TiO}_{2}$ has larger lattice parameters than $\mathrm{VO}_{2}$, so films grown on $\mathrm{TiO}_{2}$ substrates are typically strained, which can alter the MIT of $\mathrm{VO}_{2}$. However, the strain in films of $80 \mathrm{~nm}$ thickness is expected to be mostly relaxed at the surface, and thus, they should behave similarly to bulk $\mathrm{VO}_{2}{ }^{25}$ The first film (film 1) was synthesized by reactive-biased target ion beam deposition as reported previously. ${ }^{25,26}$ Prior to XPS experiments, resistivity measurements showed that the film underwent a metal-insulator transition around $60-80^{\circ} \mathrm{C}$, which is characteristic of bulk $\mathrm{VO}_{2}$, and the film was determined by XRD to be monoclinic $\mathrm{VO}_{2}$ with a small $\mathrm{V}_{2} \mathrm{O}_{5}$ component [supplementary material, Fig. S. 1.(a)]. Carbon contamination was removed by annealing at $450{ }^{\circ} \mathrm{C}$ for $30 \mathrm{~min}$ in $10^{-7}-10^{-6}$ Torr $\mathrm{O}_{2}$ prior to water adsorption experiments. Experiments were repeated on a second film (film 2). Film 2 was synthesized by pulsed laser deposition (PLD) - the details of which can be found in the supplementary material-and verified to be $\mathrm{VO}_{2}$ by XRD [Fig. S. 1.(b)]. In this case, the film was sputtered for $5 \mathrm{~min}$ to remove contamination, with a current of $2 \mu \mathrm{A}$, at $2 \mathrm{~V}$, under $5 \times 10^{-5}$ Torr Ar. This process caused the surface to be reduced, and then the film was annealed by gradually increasing the temperature up to $300{ }^{\circ} \mathrm{C}$ in $1 \times 10^{-6}-1 \times 10^{-5}$ Torr oxygen to restore the original $\mathrm{V}^{4+}$ composition, as determined from the V $2 p$ and valence band spectra (Fig. S. 2). The results from both the films are presented here in order to assess the degree to which minor variations in surface composition affect the water adsorption behavior. Milli-Q purified water (18.2 $\mathrm{M} \Omega$ $\mathrm{cm}$ ) was mounted on the APXPS analysis chamber and degassed by three cycles of liquid nitrogen freeze-pump-thaw, before being introduced into the chamber with a high precision leak valve. $\mathrm{V}$ $2 p / \mathrm{O} 1 s$ spectra were collected at an excitation energy of $735 \mathrm{eV}$, $\mathrm{C} 1 s$ at $490 \mathrm{eV}$, and the valence band at $300 \mathrm{eV}$. These energies were chosen so that all photoelectrons had a kinetic energy $\left(\mathrm{E}_{K}\right)$ of $200-300 \mathrm{eV}$, and thus originated from up to approximately $0.7-$ $0.9 \mathrm{~nm}$ within the sample. ${ }^{27}$ The Au $4 f$ spectrum of a gold foil in electrical contact with the film was measured periodically at the respective excitation energies and used to calibrate the binding energy of the other spectra, by fixing the Au $4 f_{7 / 2}$ binding energy to $84.0 \mathrm{eV}$.

\section{RESULTS}

\section{A. Fitting of APXPS spectra and identification of surface species}

Representative O $1 s$ and V $2 p$ XPS data for isotherm $\left(23^{\circ} \mathrm{C}\right)$ experiments on the two vanadium oxide films are shown in Fig. 1(a). When calibrated to the $\mathrm{Au} 4 f$ reference, the binding energy of the $\mathrm{O} 1 s$ peak is around $530.1 \mathrm{eV}( \pm 0.1 \mathrm{eV})$. The $\mathrm{V} 2 p$ region 

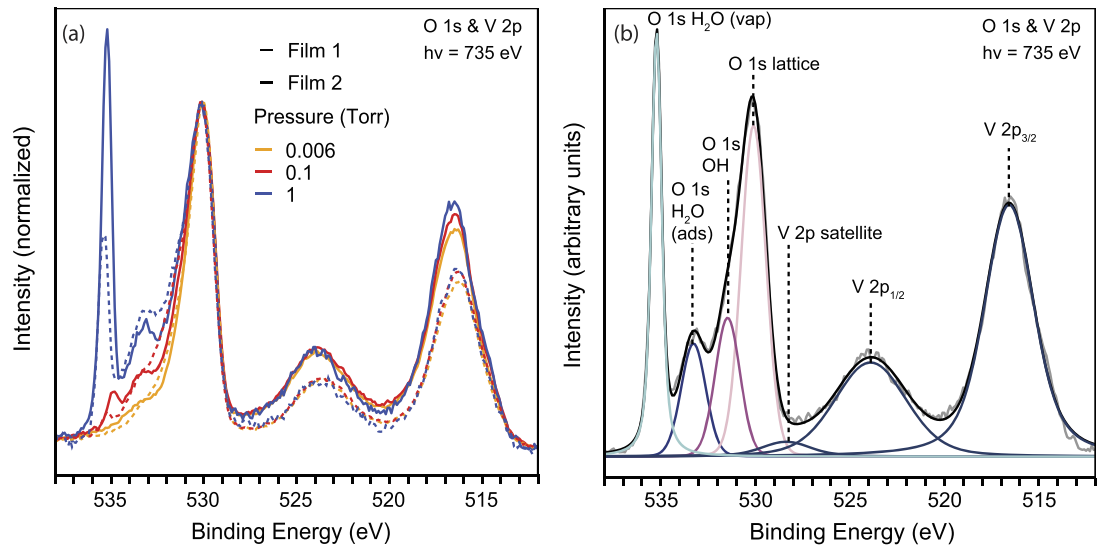

FIG. 1. (a) $01 s$ and $V 2 p$ region taken at selected water vapor pressures as part of a $23^{\circ} \mathrm{C}$ isotherm experiment for film 1 and film 2. Spectra are background subtracted and normalized to the lattice $01 \mathrm{~s}$ intensity. Solid line is film 2, and dotted line is film 1. (b) Example fit for the 0 $1 s$ and $V 2 p$ region of vanadium oxide films. This spectrum was taken on film 2 , at $23^{\circ} \mathrm{C}$, under $1 \mathrm{Torr} \mathrm{H}_{2} \mathrm{O}$, as part of an isotherm experiment.

consists of spin-orbit split V $2 p_{3 / 2}$ and $\mathrm{V} 2 p_{1 / 2}$ peaks at $516.4 \mathrm{eV}$ and $523.7 \mathrm{eV}$, respectively $( \pm 0.1 \mathrm{eV})$, which correspond roughly to the values reported for $\mathrm{V}^{4+}$ in the literature. ${ }^{28,29}$ At increasing relative humidity $(\mathrm{RH})$, the relative intensity of at least two additional peaks with higher binding energy increases in the $\mathrm{O} 1 \mathrm{~s}$ region. The peak at approximately $531.5 \mathrm{eV}$ is in the position expected for surface $-\mathrm{OH}$ species, while the peak at approximately $533 \mathrm{eV}$ is in the position expected for surface adsorbed molecular water. ${ }^{18,30,31}$ The peak at approximately $535 \mathrm{eV}$ is due to free water vapor in the chamber. A representative example of the fit is shown in Fig. 1(b). The $\mathrm{O} 1 \mathrm{~s}$ and V $2 p$ spectra are each fit as a single region, with a Shirley background. The $\mathrm{V} 2 p$ peaks are constrained to have an area ratio of 2:1 and a separation of $7.33 \mathrm{eV}$, as reported in the literature. ${ }^{28,29}$ The peak widths of the $\mathrm{V} 2 p$ peaks were not constrained to be equal since the $\mathrm{V} 2 p_{1 / 2}$ peak is broadened due to the Coster-Kronig effect. ${ }^{29} \mathrm{~A} V 2 p_{3 / 2}$ satellite was fit between the $\mathrm{V} 2 p_{1 / 2}$ and $\mathrm{O} 1 s$ peaks and constrained to be between 0.05 and 0.15 times the area of the main $\mathrm{V} 2 p_{3 / 2}$ peak, and $11.8 \mathrm{eV}$ higher binding energy, based on fits in previous studies. ${ }^{28}$ Surface O $1 s$ atoms were constrained to have the same peak width. A detailed summary of peak fitting parameters can be found in the supplementary material (Table 1).

For simplicity, each of the vanadium peaks was fit with a single Voigt function. However, it is likely that small quantities of $\mathrm{V}^{3+}$ and $\mathrm{V}^{5+}$ are present in these films. In addition to evidence of $\mathrm{V}_{2} \mathrm{O}_{5}$ in film 1 from XRD taken ex situ [Fig. S. 1.(a)], non-stoichiometry is suggested by the shapes of the V $2 p$ peaks in XPS, which are slightly different when comparing the spectra taken at the lowest and highest RH (Fig. S. 5), and when comparing the two different films. There are slight changes in intensity toward the higher and lower binding energy sides, suggesting that varying levels of $\mathrm{V}^{3+}$ and $\mathrm{V}^{5+}$ are present, but these changes are too small to be reliably captured by fitting with additional peaks. Theoretical studies have shown that the V $2 p$ peaks are actually composed of several multiplet components, so it is also possible that the changes are due to alterations in the multiplet structure, rather than in the oxidation state. ${ }^{32}$ Here, the main interest is in changes in the $\mathrm{O} 1 \mathrm{~s}$ region, so fitting the $\mathrm{V} 2 p$ region to this level of precision is beyond the scope of this study. However, minimal attention is directed to these intricacies in most publications that include V $2 p$ XPS fitting. It is of benefit to note here, the complexity of vanadium oxide spectra and highlight that there is still a lot of theoretical and experimental work to be done before XPS can reliably be used to determine the exact composition of vanadium oxide surfaces with mixed oxidation states.

One strategy used by other researchers faced with this issue was to determine the splitting between the $\mathrm{V} 2 p_{3 / 2}$ and lattice $\mathrm{O}$ $1 s$ peaks as a function of the oxidation state. A linear relationship was established: $\mathrm{V}_{o x}=13.82-0.68\left[\mathrm{O} 1 s-\mathrm{V} 2 p_{3 / 2}\right]$ (where $\mathrm{O} 1 s$ and $\mathrm{V} 2 p_{3 / 2}$ are the positions of the respective peaks in $\left.\mathrm{eV}\right) .^{33}$ The splitting between the peaks for film 1 ranged from $13.53 \mathrm{eV}$ to $13.81 \mathrm{eV}$ and for film 2 from $13.49 \mathrm{eV}$ to $13.67 \mathrm{eV}$; therefore, according to the established relationship, the average surface oxidation state varied between $4.40^{+}-4.65^{+}$and $4.55^{+}-4.65^{+}$, respectively. This suggests that in all experiments, the $\mathrm{VO}_{2}$ surface is partially oxidized to $\mathrm{V}^{5+}$. As shown in the Fig. S. 6., most isobar experiments showed a slight (0.05-0.1 charge unit) decrease in the oxidation state with increased relative humidity. However, this is not true for all experiments, and there is a lot of variation in these estimates, possibly due to inhomogeneity in the samples where some areas of the film are more oxidized than others. The isotherms do not show the same trend and generally show a higher estimated oxidation state at higher $\mathrm{RH}$. The differences between isobar and isotherm experiments suggest that there is a temperature-dependent reaction occurring. One possibility is that the sample is reversibly oxidized at the higher temperature and reduced as it cools down.

According to survey spectra (Fig. S. 4), the main impurities present on these films are carbon and potassium. The presence of carbon is expected and an established annealing method was used to remove it from the surface of film $1 .{ }^{34}$ The $\mathrm{C} 1 \mathrm{~s}$ spectra showed that this annealing procedure removed most of the carbon; however, a fairly large $\mathrm{K} 2 p$ component remained in the same region (Fig. 2). Because of this observation, additional films were grown by PLD and different methods were tested in order to remove contamination. If the films were sputtered until there was no intensity in the $\mathrm{C} 1 \mathrm{~s} / \mathrm{K} 2 p$ region, and then treated to the original annealing procedure, the $\mathrm{K} 2 p$ peaks were observed to grow at temperatures above $350^{\circ} \mathrm{C}$. It was thus established that potassium was not just surface contamination and that it was migrating to the surface of the film during heating. The source of this contamination is not clear, but since it is present on films grown by two 

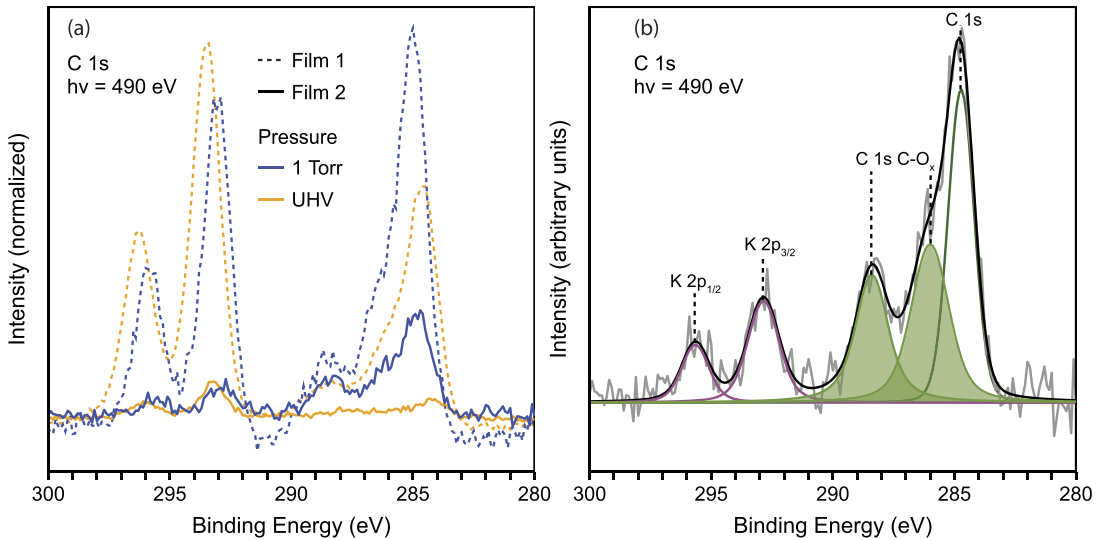

FIG. 2. (a) Comparison of $C 1 s / K 2 p$ binding energy region at the beginning and end of a $23^{\circ} \mathrm{C}$ isotherm experiment, for film 1 and film 2. Data are normalized to $01 \mathrm{~s}$ intensity collected under the equivalent conditions. Solid line is film 2 and the dotted line is film 1. (b) Example fit of the $C 1 s / K 2 p$ binding energy region taken on film 2 , at $23^{\circ} \mathrm{C}$, under 1 Torr $\mathrm{H}_{2} \mathrm{O}$ as part of an isotherm experiment. Spectra were fit with a linear background and Voigt functions.

different methods, and is not on other surfaces measured at the same beamline endstation, a likely source is the surface of the $\mathrm{TiO}_{2}$ substrates. A new preparation procedure was established, where mild sputtering was used to remove contamination and annealing in $1 \times 10^{-6}-1 \times 10^{-5}$ Torr oxygen at temperatures below $350{ }^{\circ} \mathrm{C}$ was employed to restore the original majority $\mathrm{V}^{4+}$ oxidation state. This was achieved by monitoring the $\mathrm{V} 2 p$ and valence band in situ during annealing, and halting the annealing once the spectra had returned to their original shape (Fig. S. 2). Both carbon and potassium were present on the resulting surface, but the surface concentration of potassium estimated from the relative intensity of $\mathrm{O} 1 s$ and $\mathrm{K} 2 p$ peaks was an order of magnitude lower on the sputtered film 2 surface compared to the annealed film 1 surface (see supplementary material, Sec. I). Since the surface structures resulting from these two treatments are unknown, data from films prepared by both the methods are included here in order to gain an idea of how important surface structure and impurity concentration are to the water adsorption behavior.

\section{B. Comparison of isobar and isotherm experiments}

The RH at the film surface can be controlled at constant temperature by changing the pressure and at constant pressure by changing the temperature. Both of these methods have advantages and disadvantages.

Isotherm experiments allow determination of the water adsorption process independently of the temperature and the potential phase changes that might occur as a function of temperature. This is an important consideration for vanadium oxides, which have a very complex bulk phase diagram with many intermediate phases, where multiple oxidation states coexist and a variety of temperaturedependent electronic and structural changes. $\mathrm{VO}_{2}$ in particular undergoes a change around $60-80{ }^{\circ} \mathrm{C}$, where it transforms with increased temperature from a metal to a semiconductor and from a monoclinic to a rutile structure. ${ }^{35}$ There is some evidence that this insulator-metal transition occurs in the films studied here, in the form of changes to the leading edge of the valence band (Fig. S. 3). However, this was not consistently observed after annealing or sputtering the films. Since $\mathrm{VO}_{2}$ can be transformed into a metal by various means, including trace level doping, it is possible that the loss of the transition could be related to the presence of potassium in the material, or to surface defects formed during heating or sputtering. Isotherm experiments performed at a constant temperature of $23{ }^{\circ} \mathrm{C}$ ensure that the water adsorption process is not affected by this transition.

At $23^{\circ} \mathrm{C}$, it is very difficult to achieve a clean surface, as there are trace levels of organic contamination in the XPS chamber, which can condense on the surface at this low temperature. This means that at the low $\mathrm{RH}, \mathrm{C}-\mathrm{Ox}$ species from carbon contaminants can contribute intensity to the binding energy region at 531-534 eV, and interfere with the coverage determination for $-\mathrm{OH}$ and molecular water species. In isobar experiments, low $\mathrm{RH}$ is instead achieved by heating the film to a high temperature, allowing the beginning of water adsorption to be observed on a relatively clean surface. Therefore, performing both isotherm and isobar experiments can help clarify which effects are due to $\mathrm{RH}$ and which are due to temperature or contamination. If isotherm and isobar experiments yield similar results, this also indicates that the surface and gas phase are in thermodynamic equilibrium.

Figure 3 shows the signal ratio of $-\mathrm{OH}$ and molecular water peaks to the lattice oxygen peak, as a function of $\mathrm{RH}$, for all isotherm and isobar experiments on $\mathrm{VO}_{2} / \mathrm{TiO}_{2}$ (100) films. The isotherm measurements show that as $\mathrm{RH}$ is increased, the amount of $-\mathrm{OH}$ and adsorbed water on the vanadium oxide surface increases. A more rapid increase is seen above around $0.01 \% \mathrm{RH}$. As $\mathrm{RH}$ is decreased, coverage of adsorbed water decreases, while the $-\mathrm{OH}$ coverage increases initially. At equivalent $\mathrm{RH}$, the amount of adsorbed water is higher for the decreasing isotherm.

The isobar measurements also show an increase in adsorbed water coverage with increasing $\mathrm{RH}$ with an onset of around $0.01 \%$ $\mathrm{RH}$. Generally, at equivalent $\mathrm{RH}$, the amount of adsorbed water is higher for the reverse isobar. The trends for $-\mathrm{OH}$ are less consistent. Generally, as seen in the isotherm data, $-\mathrm{OH}$ coverage increases with increasing $\mathrm{RH}$, and sometimes shows an initial increase with decreasing $\mathrm{RH}$, before decreasing. This initial increase, and the generally higher levels of adsorbates in the reverse isotherm and isobar experiments, could be occurring because an existing layer of molecular water and -OH facilitates further water adsorption and dissociation, through cooperative effects. It could also be due to changes 


\section{Symbol}

Increasing RH: Decreasing $\mathrm{RH}$ : Increasing $\mathrm{RH}, 2$ 2nd experiment:

$\diamond$ Film $1 \bullet$ Film $2 \diamond$ Film 1 O Film $2 \oplus$ Film $1 \oplus$ Film 2

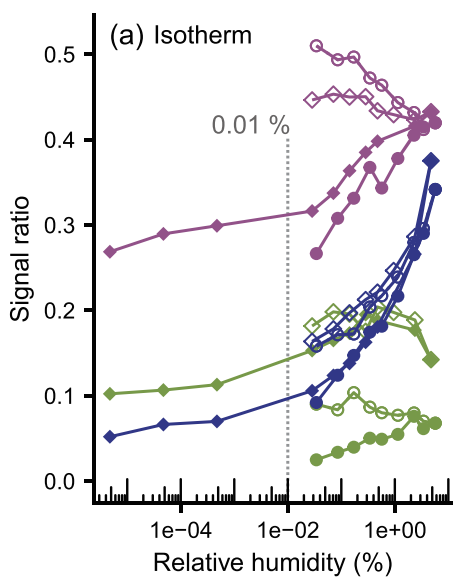

(b) Isobar 0.1 Torr

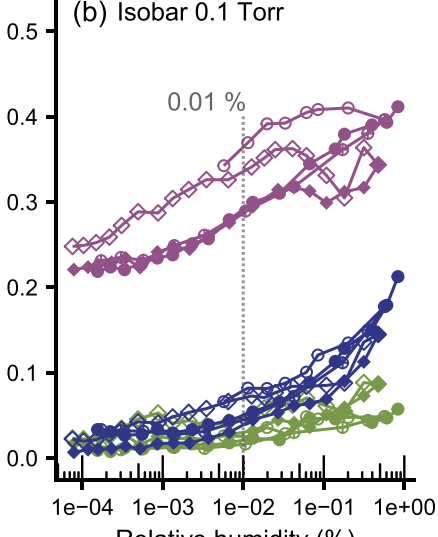

Color

$\rightarrow$ OH:O lat

$\rightarrow$ O mol: O lat

$\rightarrow$ C-Ox:O lat

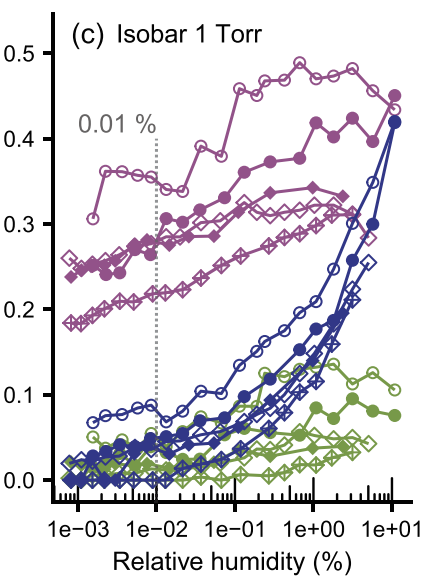

FIG. 3. Signal ratio of $-\mathrm{OH}$ peak (pink) and molecular water peak (blue) to lattice oxygen peak, along with the estimated relative $\mathrm{O} 1 \mathrm{~s}$ signal due to $\mathrm{C}-\mathrm{Ox}$ (green), as a function of $\mathrm{RH}$, for (a) isotherm experiment at $23^{\circ} \mathrm{C}$, (b) isobar experiment at $0.1 \mathrm{Torr}$, and (c) isobar experiment at 1 Torr. Onset $\mathrm{RH}$ of $0.01 \%$ is indicated by dotted line.

in the level of carbon contamination, or potentially a beam damage effect (see the supplementary material for a detailed discussion on beam damage).

As shown in Fig. 2, the carbon contamination on the surface is partially oxidized and will, therefore, contribute intensity to the $\mathrm{O} 1 s$ spectrum. The typical $\mathrm{O} 1 \mathrm{~s}$ binding energies of $\mathrm{C}-\mathrm{Ox}$ species overlap with those of the $-\mathrm{OH}$ and water peaks, so the $\mathrm{C}-\mathrm{Ox}$ contribution to the $\mathrm{O} 1 s$ spectrum cannot be determined directly by fitting additional peaks in this region. ${ }^{36}$ Using the C:O sensitivity factor determined from gas phase $\mathrm{CO}_{2}$ XPS, the minimum $\mathrm{O} 1 \mathrm{~s}$ signal intensity due to $\mathrm{C}-\mathrm{Ox}$ was estimated from the $\mathrm{C} 1 \mathrm{~s}$ spectrum (see the supplementary material for details), and is included in Fig. 3. For all experiments, this estimated $\mathrm{C}-\mathrm{Ox} \mathrm{O} 1 s$ intensity was found to be large enough to account for the peak intensity assigned to molecular water at low $\mathrm{RH}$, but does not increase as much above $0.01 \% \mathrm{RH}$, so the increase here can be attributed to the adsorption of molecular water. The $-\mathrm{OH}$ peak intensity at low $\mathrm{RH}$ is much greater than the estimated C-Ox intensity, so it can be confirmed that $-\mathrm{OH}$ is present on the surface, even at very low RH.

Comparing the same experiments on the different films, the adsorption trends are very similar for the isotherm experiments. For the isobar experiments, there is less change in the relative intensity of the $-\mathrm{OH}$ peak for film 1 compared to film 2 . This could be related to the number of adsorption sites available for $-\mathrm{OH}$, which may be influenced by potassium contamination levels or surface defects.

Figure 4 shows the estimated coverages in monolayers (see the supplementary material) as a function of $\mathrm{RH}$ for all experiments on $\mathrm{VO}_{2} / \mathrm{TiO}_{2}(100)$. There is a lot of spread in the data, but some rough overall trends can be established. The relative intensity of the molecular water peak remains fairly constant up to $0.01 \% \mathrm{RH}$ and then between $0.01 \%$ and $0.1 \%$ starts to increase sharply. The coverage of molecular water grows more rapidly than the coverage of $-\mathrm{OH}$ so that the coverages become equal at approximately $10 \% \mathrm{RH}$, with a total coverage of $>1 \mathrm{ML}$. At low $\mathrm{RH}$, there is a minimum of 0.3-0.5 ML $-\mathrm{OH}$, which typically forms at surface defects on metal

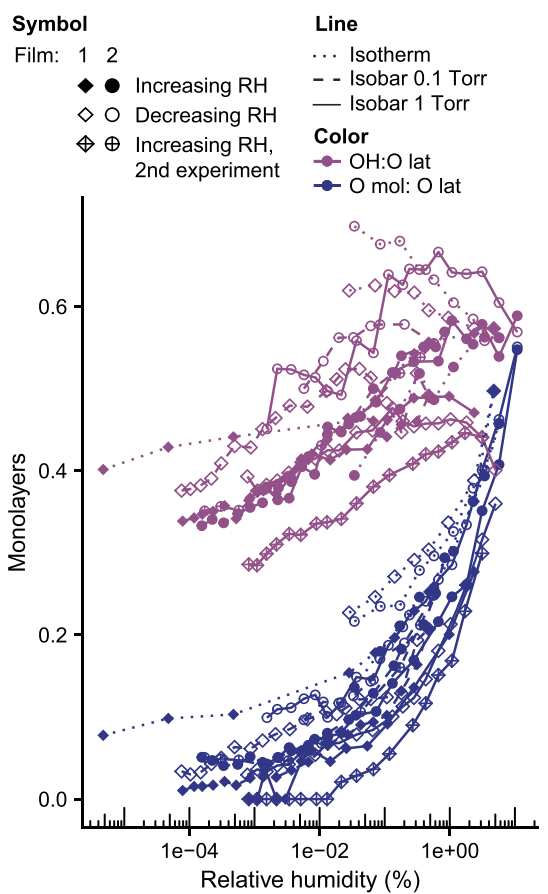

FIG. 4. Coverage in monolayers of $-\mathrm{OH}$ and molecular $\mathrm{H}_{2} \mathrm{O}(\mathrm{O} \mathrm{mol})$ from all isotherm and isobar experiments plotted on the same axis. 
oxides at low relative humidity. However, $10 \%-50 \%$ of this could be $\mathrm{C}-\mathrm{Ox}$, depending on what carbon species are present. Molecular water coverage below $0.01 \% \mathrm{RH}$ is approximately $0.1 \mathrm{ML}$, but up to $100 \%$ of this peak intensity could be due to $\mathrm{C}-\mathrm{Ox}$, so $0.01 \% \mathrm{RH}$ is considered the adsorption onset for molecular water.

\section{Work function and valence band}

The position of the water vapor peak relative to other peaks is observed to change with $\mathrm{RH}$. The apparent binding energy is observed to increase (i.e., its kinetic energy decreases) by up to $1 \mathrm{eV}$ as $\mathrm{RH}$ is increased (Fig. 5). This can also be seen for the water vapor peaks observed in the valence band region [Fig. 6(a)]. The vacuum level of gas molecules in the vicinity of a solid film's surface is determined by the material surface work function. A change of the vacuum level relative to the Fermi level must induce a corresponding change in the detected apparent binding energy of the gas phase core level photoelectrons. Therefore, a change in the apparent binding energy of the vapor peak can be related to a change in the work function of the material. ${ }^{1}$ The work function and binding energy are negatively correlated, so it appears that the work function decreases with RH.

The degree to which this occurs is greater for film 2 than for film 1 , with binding energies up to $0.5 \mathrm{eV}$ lower on film 2 at the lowest RH [Fig. 5(b)]. This suggests that the films have a different work function in the absence of water. However, the change of the gas phase binding energy is also geometry dependent, so the difference between films could be due to different distances from the film to detector aperture. This is supported also by the different intensity of the water vapor peak relative to lattice oxygen peak for the two different films. It is important to point out that the qualitative trend is, however, the same for both films.

The work function changes can be induced by electron transfer between surfaces and adsorbates. The changes in work function follow a similar pattern as the molecular water coverage, with a change in slope at approximately $0.01 \% \mathrm{RH}$, suggesting that these properties are closely related. A decrease in work function can result from the presence of a surface dipole layer, in which the positive charge is directed away from the substrate. This trend is consistent with adsorption of $\mathrm{H}_{2} \mathrm{O}$ (or $\mathrm{OH}$ ) through the (negatively charged) oxygen atom with the (positively charged) hydrogen atoms away from the surface. ${ }^{17,31}$ Similar observations have been made in low-temperature UHV studies of water adsorption on $\mathrm{V}_{2} \mathrm{O}_{3}$, where the work function was found to decrease with increasing $\mathrm{H}_{2} \mathrm{O}$ exposure.

The valence band consists of a component at $0-2.5 \mathrm{eV}$, which is attributed to pure $\mathrm{V} 3 d$ states, and a component at $2.5-9 \mathrm{eV}$, which has mostly $\mathrm{O} 2 p$ character. ${ }^{37}$ When water is introduced, three additional components appear at approximately $7 \mathrm{eV}, 10 \mathrm{eV}$, and $14 \mathrm{eV}$, which correspond to the $1 b_{1}, 3 a_{1}$, and $1 b_{2}$ molecular orbitals of water, respectively. ${ }^{31}$ These show a similar shift to the $\mathrm{O} 1 \mathrm{~s}$ gas phase peak and their spacing is within the range observed for gas phase water, so these are likely predominantly from gas phase water. In most cases, the valence band shows a shift in the low energy side of the $\mathrm{O} 2 p$ states toward higher binding energy with increased RH (decreased temperature, Fig. 6). This was quantified by fitting a linear model to the side of the peak, and determining the $x$ intercept. The shift is smaller than the shift seen in the vapor peaks, not exceeding $0.5 \mathrm{eV}$, and for film 1 , some of the experiments do not show an obvious trend with RH. Band bending can be ruled out since the position of the low energy side of the $\mathrm{V} 3 d$ states does not change, and the core level peaks do not shift to the same degree. A similar shift in valence band $\mathrm{O} 2 p$ states has been observed in hydrogen adsorption studies on $\mathrm{VO}_{2}$. This is speculated to be due to relaxation of the surface upon $\mathrm{H}_{2}$ adsorption, with the small change in bond length or angle resulting in a change (increase) in $\mathrm{V}-\mathrm{O} \pi$ bonding interaction. The increased bonding character would result in a decrease in the energy of the O $2 p \pi$ band. ${ }^{38}$ Water may similarly induce relaxation through its interaction with the $\mathrm{VO}_{2}$ surface, which could also increase $\mathrm{V}-\mathrm{O}$ $2 p \pi$-orbital overlap, resulting in a similar change in the valence band.
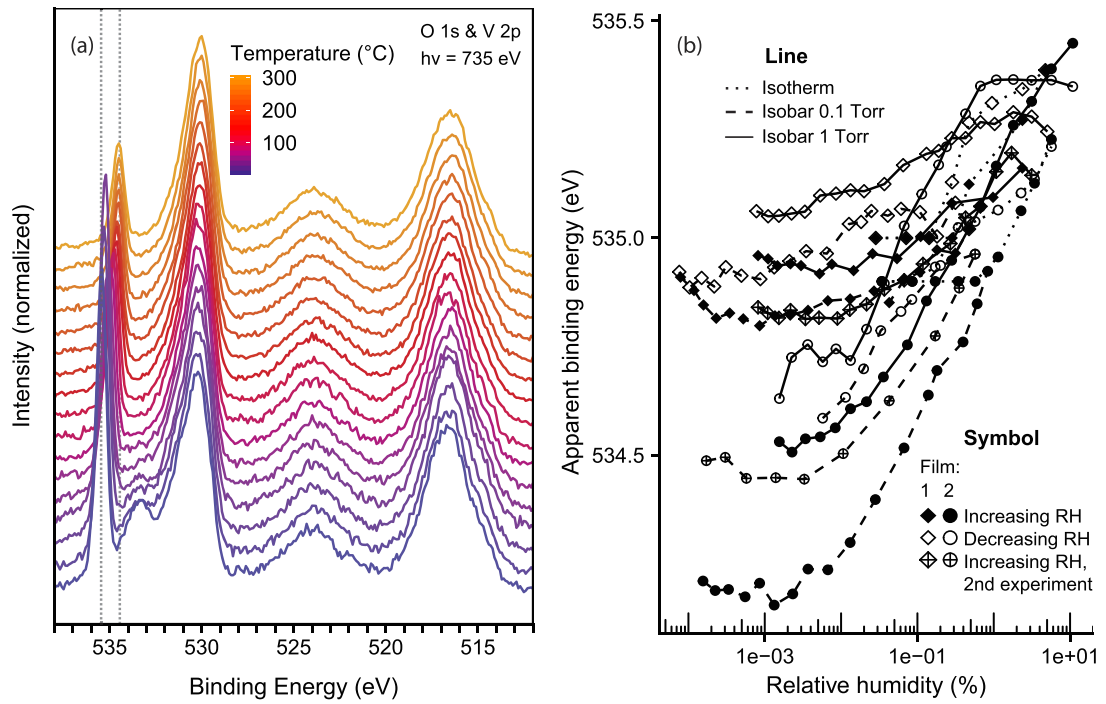

FIG. 5. (a) Spectra from 1 Torr isobar on film 2, displaying a clear shift (dotted line) in the water vapor peak position relative to other peaks as $\mathrm{RH}$ is increased (temperature is decreased). Spectra are background subtracted, normalized to the lattice $01 s$ intensity, and offset. (b) Binding energy of water vapor peak as a function of $\mathrm{RH}$ for all isotherm and isobar experiments on $\mathrm{VO}_{2} / \mathrm{TiO}_{2}(100)$. 

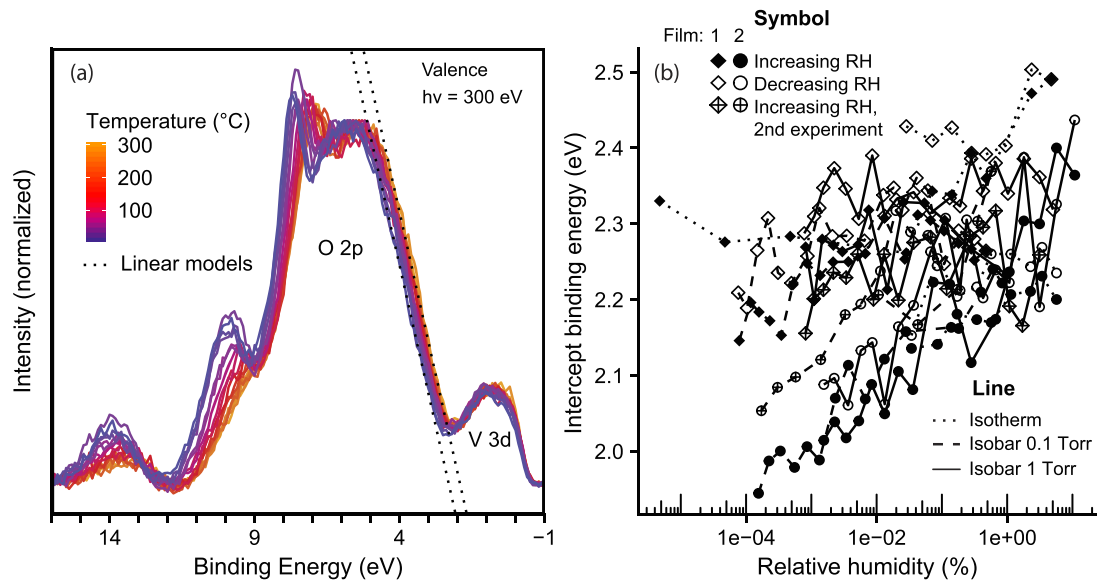

FIG. 6. (a) Spectra from 1 Torr isobar, on film 2, showing changes in the valence band with increasing $\mathrm{RH}$ (decreasing temperature), with the linear models used to represent the 0 $2 p$ position for the highest and lowest temperature (dotted lines). Spectra are background subtracted and normalized to the maximum of the $02 p$ component. (b) Binding energy, at which a linear model of the shifting $O 2 p$ edge intersects the $x$ axis, as a function of $\mathrm{RH}$ for all isotherm and isobar experiments on $\mathrm{VO}_{2} / \mathrm{TiO}_{2}(100)$.

It is interesting to note that the experiments in which the least change is observed in the $\mathrm{O} 2 p$ position are the same experiments in which little change in -OH coverage is observed (1 Torr and 0.1 Torr isobars for film 1). This suggests that the valence band shift is related to the appearance of $\mathrm{OH}$ (or C-Ox species with the same $\mathrm{O} 1 s$ binding energy). Another related observation is that in the absence of water vapor, the $\mathrm{O} 2 p$ position is correlated with the presence of a feature at approximately $10.5 \mathrm{eV}$, shifting to higher energies when the feature is present. For example, this can be seen in the spectra taken before and after cleaning, as shown in Fig. S. 3 .

The distribution of the $\mathrm{V} 3 d$ states also changes slightly, with the intensity maximum moving from around $0.7 \mathrm{eV}$ to $1.2 \mathrm{eV}$ as $\mathrm{RH}$ is increased. This suggests that the adsorbates are interacting with the $\mathrm{V} 3 d$ states and shifting their energy.

\section{DISCUSSION}

A general behavior observed in similar studies is that hydroxylation precedes water adsorption. ${ }^{1,2,39}$ It is speculated that $\mathrm{H}_{2} \mathrm{O}-\mathrm{OH}$ complexes make layers containing $\mathrm{OH}$ more stable than pure layers of molecular water. This stability originates from the $\mathrm{H}_{2} \mathrm{O}-\mathrm{OH}$ hydrogen bonds, which are approximately $0.18 \mathrm{eV}$ stronger than the $\mathrm{H}_{2} \mathrm{O}-\mathrm{H}_{2} \mathrm{O}$ hydrogen bonds. ${ }^{39}$ Additionally, theoretical studies indicate that dissociation of an isolated water molecule is kinetically hindered, while dissociation of a water molecule that forms a complex with other adsorbed water molecules, as well as lattice oxygen, is more facile. ${ }^{40,41} \mathrm{~A} \mathrm{RH}$ of $0.01 \%$ has been identified as the critical onset value for the formation of these complexes. ${ }^{42,43}$ On the $\mathrm{VO}_{2}$ surface presented in this work, the presence of $-\mathrm{OH}$ precedes $\mathrm{H}_{2} \mathrm{O}$ adsorption and in most cases coverage of both species begins to increase more rapidly around $0.01 \% \mathrm{RH}$, so $\mathrm{VO}_{2}$ is consistent with the proposed model.

The difference in the kinetic barrier for $-\mathrm{OH}$ formation and the number and nature of $-\mathrm{OH}$ groups on surfaces can be related to defect concentration. ${ }^{39}$ For example, in similar studies on $\mathrm{TiO}_{2}$, a small concentration of O-vacancy defects in the rows of bridging oxygen atoms $(0.125 \mathrm{ML})$ was found to always be present after surface preparation. Hydroxylation on $\mathrm{TiO}_{2}$ has been found to occur only at the oxygen vacancies with coverage reaching saturation at twice the initial defect concentration $(0.25 \mathrm{ML})$. Once $-\mathrm{OH}$ is present on $\mathrm{TiO}_{2}, \mathrm{H}_{2} \mathrm{O}$ coverage rises gradually as $\mathrm{RH}$ is increased, and starts to increase more rapidly once it equals $\mathrm{OH}$ coverage. ${ }^{44}$ On $\mathrm{VO}_{2}$, unlike $\mathrm{TiO}_{2}$, it is not straightforward to identify oxygen defects by XPS, due to the complicated nature of the vanadium core levels. However, it is likely that some kinds of defects are present. It is possible that these defects are filled by dissociative adsorption of water to form the observed initial $-\mathrm{OH}$ coverage of $0.3-$ $0.5 \mathrm{ML}$. If each defect is forming two -OH groups, defect levels would be 1-2 times the concentration observed on the $\mathrm{TiO}_{2}$ surface, which seems reasonable considering the contribution of $\mathrm{C}-\mathrm{Ox}$ to the $-\mathrm{OH}$ signal, and the less well-refined surface preparation procedures used here. In contrast to $\mathrm{TiO}_{2},-\mathrm{OH}$ coverage on $\mathrm{VO}_{2}$ seems to increase simultaneously with the onset of $\mathrm{H}_{2} \mathrm{O}$ adsorption in most cases, suggesting that dissociation is not limited to defect sites on this surface. At the same $\mathrm{RH}$, the $\mathrm{H}_{2} \mathrm{O}$ coverage is lower on $\mathrm{VO}_{2}$ than on $\mathrm{TiO}_{2}$ and unlike on $\mathrm{TiO}_{2}$, it does not exceed $\mathrm{OH}$ coverage in this $\mathrm{RH}$ range. One possible explanation for this phenomenon is that the $\mathrm{H}_{2} \mathrm{O}$ coverage is limited by its dissociation into $\mathrm{OH}$. In another example, on $\mathrm{MgO}$ and $\alpha-\mathrm{Fe}_{2} \mathrm{O}_{3}, \mathrm{OH}$ coverage is observed to increase at a similar $0.01 \% \mathrm{RH}$ threshold, and then saturate at $1 \mathrm{ML}^{43}$ It would be interesting in future studies to examine the $\mathrm{VO}_{2}$ surface at higher $\mathrm{RH}$ to determine if such a saturation point is reached.

This study can be related to a recent APXPS study of water adsorption on polycrystalline vanadium metal. ${ }^{20}$ In the presence of water above $1 \times 10^{-6} \% \mathrm{RH}$, vanadium metal was found to oxidize to $\mathrm{V}_{2} \mathrm{O}_{3}$ with $\mathrm{V}-\mathrm{OH}$ on the surface. In isobar experiments starting at $1 \times 10^{-4} \% \mathrm{RH}$, the $-\mathrm{OH}$ coverage was initially around $1 \mathrm{ML}$ and increased steadily to $1.5 \mathrm{ML}$ at $15 \% \mathrm{RH}$. The molecular water coverage on this metal derived surface was relatively low compared to the $\mathrm{VO}_{2}$ surface studied here, reaching a coverage of only $1 / 3 \mathrm{ML}$ at $10 \% \mathrm{RH}$. It was observed to start growing gradually at $0.001 \% \mathrm{RH}$ and then more rapidly at $0.5 \% \mathrm{RH}$. The slower uptake and lower levels of $\mathrm{H}_{2} \mathrm{O}$ on the metal-derived surface suggest that the more oxidized and less hydroxylated surface studied in this work is more favorable for water adsorption than the 
hydroxide-terminated $\mathrm{V}_{2} \mathrm{O}_{3}$ surface derived from vanadium metal. Previous IR and UHV XPS investigations of $\mathrm{H}_{2} \mathrm{O}$ dosed on $\mathrm{V}_{2} \mathrm{O}_{3}$ surfaces have indicated that the amount of adsorbed water strongly depends on the surface termination of $\mathrm{V}_{2} \mathrm{O}_{3}$, with more water adsorption observed on vanadium-terminated surfaces. Vanadylterminated surfaces were less active toward water adsorption. ${ }^{18}$ Similarly, the hydroxyl groups formed on the metal-derived surface could be acting as a passivating layer, while on the surface in our study there may be more under-coordinated surface vanadium.

\section{CONCLUSION}

This study has shown that the onset for molecular water adsorption on these $\mathrm{VO}_{2} / \mathrm{TiO}_{2}(100)$ films is preceded by $\mathrm{OH}$ formation and consistently occurs at approximately $0.01 \% \mathrm{RH}$, independent of temperature, differences in potassium and carbon contamination levels, and differences in the surface preparation method. This is consistent with the general trends seen on oxide surfaces and can be related to the formation of hydrogen bonded surface complexes. The work function changes suggest that the adsorbates are bound with the hydrogen atoms pointing away from the surface. Changes in the valence band suggest that the adsorbates have some influence on the surface electronic structure, possibly altering the $\mathrm{V}-\mathrm{O}$ bond lengths and angles, and interacting with the $\mathrm{V} 3 d$ states. This oxide surface shows different water adsorption properties compared to vanadium metal, with less hydroxide forming and greater uptake of molecular water. There is still much to be understood about vanadium oxide surfaces. Additional APXPS studies on vanadium oxides of different oxidation states, phases, and surface orientations could uncover trends of interest for the design of improved catalysts, or for tuning the electronic properties of oxide-based electronic devices.

\section{SUPPLEMENTARY MATERIAL}

See the supplementary material for supporting discussions, figures and tables, including details of film synthesis and characterization, peak fitting parameters, coverage calculations, and additional spectra.

\section{ACKNOWLEDGMENTS}

This research used resources of the Advanced Light Source, which is a DOE Office of Science User Facility under Contract No. DE-AC02-05CH11231. D.G. is grateful for an ALS Doctoral Fellowship in Residence. H.B., C.B., H.H., and S.G. acknowledge support from the Division of Chemical Sciences, Geosciences and Biosciences of the U.S. Department of Energy at LBNL under Contract No. DE-AC02-05CH11231. C.B. is grateful for support from the Alexander von Humboldt Foundation through a Feodor Lynen Research Fellowship. S.G. is grateful for support from a PROMOS scholarship provided by the Freie Universität Berlin. J.B.F. acknowledges support from the European Union Horizon 2020 under the Marie Sklodowska-Curie Grant Agreement No. 705339 and is grateful for support from the Science and Technology Facilities Council Early Career Award No. ST/K00171X/1. The Boston University program was supported by the Department of Energy under Grant No. DE-FG02-98ER45680.

\section{REFERENCES}

${ }^{1}$ H. Bluhm, "Photoelectron spectroscopy of surfaces under humid conditions," J. Electron Spectrosc. Relat. Phenom. 177, 71-84 (2010).

${ }^{2}$ G. E. Brown and G. Calas, "Mineral-aqueous solution interfaces and their impact on the environment," Geochem. Perspect. 1, 483 (2013).

${ }^{3}$ V. K. Khanna, "Humidity and contamination effects on electronics," in ExtremeTemperature and Harsh-Environment Electronics (IOP Publishing, 2017), pp. 15-1 $-15-11$.

${ }^{4}$ Z. Yang, C. Ko, and S. Ramanathan, "Oxide electronics utilizing ultrafast metalinsulator transitions,” Annu. Rev. Mater. Res. 41, 337 (2011).

${ }^{5}$ Y. Ke, S. Wang, G. Liu, M. Li, T. J. White, and Y. Long, "Vanadium dioxide: The multistimuli responsive material and its applications," Small 14, 1802025 (2018).

${ }^{6}$ S. Surnev, M. G. Ramsey, and F. P. Netzer, "Vanadium oxide surface studies," Prog. Surf. Sci. 73, 117-165 (2003).

${ }^{7}$ J. Stringer, “The vanadium-oxygen system-A review," J. Less Common Met. 8, 1-14 (1965).

${ }^{8} \mathrm{U}$. Schwingenschlogl and V. Eyert, "The vanadium Magneli phases $\mathrm{V}_{n} \mathrm{O}_{2 n-1}$," Ann. Phys. 13, 475-510 (2004).

${ }^{9}$ V. E. Henrich, The Surface Science of Metal Oxides (Cambridge University Press, Cambridge, New York, 1994).

${ }^{10} \mathrm{I}$. E. Wachs, "Catalysis science of supported vanadium oxide catalysts," Dalton Trans. 42, 11762-11769 (2013).

${ }^{11}$ M. S. Wong, Metal Oxides Chemistry and Applications (CRC Press, 2006), pp. 31-54.

${ }^{12}$ B. M. Weckhuysen and D. E. Keller, "Chemistry, spectroscopy and the role of supported vanadium oxides in heterogeneous catalysis," Catal. Today 78, 25-46 (2003).

${ }^{13}$ Z. Li, J. Wu, Z. Hu, Y. Lin, Q. Chen, Y. Guo, Y. Liu, Y. Zhao, J. Peng, W. Chu, $\mathrm{C}$. $\mathrm{Wu}$, and $\mathrm{Y}$. Xie, "Imaging metal-like monoclinic phase stabilized by surface coordination effect in vanadium dioxide nanobeam," Nat. Commun. 8, 15561 (2017).

${ }^{14}$ J. Liang, W. Li, J. Liu, and M. Hu, "Room temperature $\mathrm{CH}_{4}$ sensing properties of Au decorated $\mathrm{VO}_{2}$ nanosheets," Mater. Lett. 184, 92-95 (2016).

${ }^{15}$ F. Pfuner, J. Schoiswohl, M. Sock, S. Surnev, M. G. Ramsey, and F. P. Netzer, "The metal-insulator transition in $\mathrm{V}_{2} \mathrm{O}_{3}(0001)$ thin films: Surface termination effects," J. Phys.: Condens. Matter 17, 4035 (2005).

${ }^{16}$ S. Hiroki, S. Yoshiaki, S. Noriya, N. Masaki, F. Takanori, and I. Yoshihiro, "Raising the metal-insulator transition temperature of $\mathrm{VO}_{2}$ thin films by surface adsorption of organic polar molecules," Appl. Phys. Express 8, 121101 (2015).

${ }^{17}$ D. S. Toledano, P. Metcalf, and V. E. Henrich, "Photoemission studies of $\mathrm{H}_{2} \mathrm{O}$ adsorption on pure and Cr-doped $\mathrm{V}_{2} \mathrm{O}_{3}$," Surf. Sci. 472, 21-32 (2001).

${ }^{18}$ M. Abu Haija, S. Guimond, A. Uhl, H. Kuhlenbeck, and H. J. Freund, “Adsorption of water on thin $\mathrm{V}_{2} \mathrm{O}_{3}$ (0001) films," Surf. Sci. 600, 1040-1047 (2006).

${ }^{19}$ R. L. Kurtz and V. E. Henrich, "Surface electronic structure and chemisorption on corundum transition-metal oxides: $\mathrm{V}_{2} \mathrm{O}_{3}$," Phys. Rev. B 28, 6699-6706 (1983).

${ }^{20}$ C. Rameshan, M. L. Ng, A. Shavorskiy, J. T. Newberg, and H. Bluhm, "Water adsorption on polycrystalline vanadium from ultra-high vacuum to ambient relative humidity," Surf. Sci. 641, 141-147 (2015).

${ }^{21}$ E. M. Vass, M. Hävecker, S. Zafeiratos, D. Teschner, A. Knop-Gericke, and R. Schlögl, "The role of carbon species in heterogeneous catalytic processes: An in situ soft x-ray photoelectron spectroscopy study," J. Phys.: Condens. Matter 20, 184016 (2008).

${ }^{22}$ E. Kleimenov, H. Bluhm, M. Hävecker, A. Knop-Gericke, A. Pestryakov, D. Teschner, J. A. Lopez-Sanchez, J. K. Bartley, G. J. Hutchings, and R. Schlögl, "XPS investigations of VPO catalysts under reaction conditions," Surf. Sci. 575, 181-188 (2005).

${ }^{23}$ L. Artiglia, S. Agnoli, and G. Granozzi, "Vanadium oxide nanostructures on another oxide: The viewpoint from model catalysts studies," Coord. Chem. Rev. 301-302, 106-122 (2015).

${ }^{24}$ D. Frank Ogletree, H. Bluhm, E. D. Hebenstreit, and M. Salmeron, "Photoelectron spectroscopy under ambient pressure and temperature conditions," Nucl. Instrum. Methods Phys. Res., Sect. A 601, 151-160 (2009). 
${ }^{25}$ S. Kittiwatanakul, S. A. Wolf, and J. Lu, "Large epitaxial bi-axial strain induces a Mott-like phase transition in $\mathrm{VO}_{2}$," Appl. Phys. Lett. 105, 073112 (2014); arXiv: 1406.7412

${ }^{26}$ K. G. West, J. Lu, J. Yu, D. Kirkwood, W. Chen, Y. Pei, J. Claassen, and S. A. Wolf, "Growth and characterization of vanadium dioxide thin films prepared by reactive-biased target ion beam deposition," J. Vac. Sci. Technol. A 26, 133-139 (2008).

${ }^{27}$ C. J. Powell and A. Jablonski, NIST Electron Inelastic-Mean-Free-Path Database, Version 1.2, 2010.

${ }^{28}$ G. Silversmit, D. Depla, H. Poelman, G. B. Marin, and R. De Gryse, "Determination of the V2p XPS binding energies for different vanadium oxidation states $\left(\mathrm{V}^{5+}\right.$ to $\left.\mathrm{V}^{0+}\right)$," J. Electron Spectrosc. Relat. Phenom. 135, 167-175 (2004).

${ }^{29}$ M. C. Biesinger, L. W. M. Lau, A. R. Gerson, and R. S. C. Smart, "Resolving surface chemical states in XPS analysis of first row transition metals, oxides and hydroxides: Sc, Ti, V, Cu and Zn,” Appl. Surf. Sci. 257, 887-898 (2010).

${ }^{30}$ P. A. Thiel and T. E. Madey, "The interaction of water with solid surfaces: Fundamental aspects," Surf. Sci. Rep. 7, 211-385 (1987).

${ }^{31}$ M. A. Henderson, "The interaction of water with solid surfaces: Fundamental aspects revisited," Surf. Sci. Rep. 46, 1-308 (2002).

${ }^{32}$ R. P. Gupta and S. K. Sen, "Calculation of multiplet structure of core p-vacancy levels. II,” Phys. Rev. B 12, 15-19 (1975).

${ }^{33}$ G. W. Coulston, E. A. Thompson, and N. Herron, "Characterization of VPO catalysts by X-ray photoelectron spectroscopy,” J. Catal. 163, 122-129 (1996).

${ }^{34}$ J. Laverock, A. R. H. Preston, D. Newby, K. E. Smith, S. Sallis, L. F. J. Piper, S. Kittiwatanakul, J. W. Lu, S. A. Wolf, M. Leandersson, and T. Balasubramanian, "Photoemission evidence for crossover from Peierls-like to Mott-like transition in highly strained $\mathrm{VO}_{2}$," Phys. Rev. B 86, 195124 (2012).

${ }^{35}$ A. Zylbersztejn and N. F. Mott, "Metal-insulator transition in vanadium dioxide," Phys. Rev. B 11, 4383-4395 (1975).
${ }^{36}$ G. Beamson and D. Briggs, in High Resolution XPS of Organic Polymers: The Scienta ESCA300 Database, edited by D. D. Briggs (John Wiley \& Sons Ltd., Chichester, England, 1992).

${ }^{37}$ C. Blaauw, F. Leenhouts, F. van der Woude, and G. A. Sawatzky, "The metalnon-metal transition in $\mathrm{VO}_{2}$ : X-ray photoemission and resistivity measurements," J. Phys. C: Solid State Phys. 8, 459-468 (1975).

${ }^{38}$ V. M. Bermudez, R. T. Williams, J. P. Long, R. K. Reed, and P. H. Klein, "Photoemission study of hydrogen adsorption on vanadium dioxide near the semiconductor-metal phase transition," Phys. Rev. B 45, 9266-9271 (1992).

${ }^{39} \mathrm{~S}$. Yamamoto, H. Bluhm, K. Andersson, G. Ketteler, H. Ogasawara, M. Salmeron, and A. Nilsson, "In situ x-ray photoelectron spectroscopy studies of water on metals and oxides at ambient conditions," J. Phys.: Condens. Matter 20, 184025 (2008).

${ }^{40} \mathrm{~K}$. Jug, B. Heidberg, and T. Bredow, "Molecular dynamics study of water adsorption structures on the $\mathrm{MgO}(100)$ surface," J. Phys. Chem. C 111, 6846-6851 (2007).

${ }^{41}$ K. Jug, B. Heidberg, and T. Bredow, "Cyclic cluster study of water adsorption structures on the $\mathrm{MgO}(100)$ surface," Surf. Sci. 601, 1529-1535 (2007).

${ }^{42}$ L. Trotochaud, A. R. Head, S. Pletincx, O. Karslığlu, Y. Yu, A. Waldner, L. Kyhl, T. Hauffman, H. Terryn, B. Eichhorn, and H. Bluhm, "Water adsorption and dissociation on polycrystalline copper oxides: Effects of environmental contamination and experimental protocol," J. Phys. Chem. B 122, 1000-1008 (2018).

${ }^{43}$ J. T. Newberg, D. E. Starr, S. Yamamoto, S. Kaya, T. Kendelewicz, E. R. Mysak, S. Porsgaard, M. B. Salmeron, G. E. Brown, A. Nilsson, and H. Bluhm, "Autocatalytic surface hydroxylation of $\mathrm{MgO}(100)$ terrace sites observed under ambient conditions," J. Phys. Chem. C 115, 12864-12872 (2011).

${ }^{44}$ G. Ketteler, S. Yamamoto, H. Bluhm, K. Andersson, D. E. Starr, D. F. Ogletree, H. Ogasawara, A. Nilsson, and M. Salmeron, "The nature of water nucleation sites on $\mathrm{TiO}_{2}(110)$ surfaces revealed by ambient pressure X-ray photoelectron spectroscopy," J. Phys. Chem. C 111, 8278-8282 (2007). 